\title{
Correspondence
}

Jin Zhang, Mark A. Anastasio*, Xiaochuan Pan, and Lihong V. Wang

\section{Weighted Expectation Maximization Reconstruction Algorithms for Thermoacoustic Tomography}

mathematically to the conventional EM algorithm, but generally propagate data inconsistencies in different ways. Images are reconstructed from simulated and experimental TAT measurement data to validate the proposed algorithms and corroborate our theoretical assertions.

\section{Imaging Model AND Data TRUnCATION}

In TAT, an electromagnetic pulse is used to irradiate a tissue sample and a thermoacoustic effect results in the generation of a pressure wavefield $p(\vec{r}, t)$. This pressure wavefield is measured by use of wide-band ultrasonic transducers that are located on the surface of a spherical [or circular in the two-dimensional (2-D) case] measurement aperture $\Omega_{0}$ that encloses the sample. Assuming that the object has homogeneous acoustic properties and the probing elec- tromagnetic pulse has a negligible temporal width, the pressure wavefield $p\left(\vec{r}_{0}, t\right)$ that is measured as a function of time $t$ at transducer location $\vec{r}_{0} \in \Omega_{0}$ can be expresses as [4]

$$
p\left(\vec{r}_{0}, t_{0}\right)=\eta \int d^{3} \vec{r} A(\vec{r}) \frac{d}{d t_{0}} \frac{\delta\left(t_{0}-\frac{\left|\vec{r}_{0}-\vec{r}\right|}{c_{0}}\right)}{4 \pi\left|\vec{r}_{0}-\vec{r}\right|}
$$

Thermoacoustic tomography is an important emerging imaging technique with potential for a wide range of biomedical imaging applications [1]. Previously, we proposed and investigated reconstruction methods for TAT that were based on the half-time reflectivity tomography paradigm [2], [3]. These reconstruction methods permit accurate image reconstruction from knowledge of only half of the detected pressure signal at each location on the receiving aperture. This is possible because a complete set of TAT measurement data contains twice as much information as is required theoretically for stable image reconstruction.

Reconstruction algorithms for TAT that are in current use assume that the object is acoustically homogeneous. An attractive characteristic of the half-time reconstruction approaches is that they can mitigate image artifacts due to heterogeneous acoustic properties of an object [2]. However, an undesirable feature of the half-time reconstruction approaches is that they can produce artifacts in the central region of image space that are attributable to the abrupt truncation of the data function. Such artifacts can obscure centrally located object features and, in some cases, hinder the diagnostic interpretation of the images.

In this correspondence, an infinite family of weighted expectation maximization (EM) algorithms is proposed for reconstructing images from temporally truncated TAT measurement data. We demonstrate that suitable choices of the weighted EM algorithm can mitigate image artifacts that are attributable to temporal truncation of the TAT data function. The weighted EM algorithms are shown to be equivalent

Manuscript received September 29, 2004; revised March 23, 2005. The Associate Editor responsible for coordinating the review of this paper and recommending its publication was M. Insana. Asterisk indicates corresponding author.

J. Zhang is with the Department of Biomedical Engineering, Illinois Institute of Technology, Chicago, IL 60616 USA.

*M. A. Anastasio is with the Department of Biomedical Engineering, Illinois Institute of Technology, 10 W. 32nd St., E1-116, Chicago, IL 60616 USA (e-mail: anastasio@iit.edu).

X. Pan is with the Department of Radiology, The University of Chicago, Chicago, IL 60637 USA.

L. V. Wang is with the Department of Biomedical Engineering, Texas A\&M University, College Station, TX 77843 USA

Digital Object Identifier 10.1109/TMI.2005.848372 where $A(\vec{r})$ denotes the spatial energy absorption function that resides inside a sphere of radius $R_{A}, c_{0}$ is the (constant) velocity of sound in the object and background medium, and the constant $\eta$ represents the isobaric volume expansion coefficient divided by the specific heat of the medium. The reconstruction problem of TAT [4], [5] is to invert (1) for determination of $A(\vec{r})$ from knowledge of $p\left(\vec{r}_{0}, t_{0}\right)$ for $\vec{r}_{0} \in \Omega_{0}$ and $t_{0} \in\left(0,\left|\vec{r}_{0}\right| / c_{0}+R_{A} / c_{0}\right]$.

The TAT data function and the spherical Radon transform are related as [2]

$$
g\left(\vec{r}_{0}, \bar{t}\right) \equiv \frac{4 \pi}{\eta} t \int_{0}^{t} d t_{0} p\left(\vec{r}_{0}, t_{0}\right)=\int d^{3} \vec{r} A(\vec{r}) \delta\left(\bar{t}-\left|\vec{r}_{0}-\vec{r}\right|\right)
$$

where $\bar{t} \equiv c_{0} t$ and $g\left(\vec{r}_{0}, \bar{t}\right)$ can be interpreted as a spherical Radon transform of $A(\vec{r})$. Equation (2) has the same mathematical form as the imaging model in reflectivity tomography [6], [7]. Consequently, image reconstruction in TAT can be accomplished by inverting the spherical Radon transform in (2) via a reflectivity tomography reconstruction algorithm.

Existing reflectivity tomography reconstruction algorithms [6]-[8] assume that $g\left(\vec{r}_{0}, \bar{t}\right)$ is untruncated with respect to $\bar{t}$. However, it has been demonstrated [3], [8] that knowledge of $g\left(\vec{r}_{0}, \bar{t}\right)$ for $\vec{r}_{0} \in \Omega_{0}$ and $\bar{t} \in\left(0,\left|\vec{r}_{0}\right|+\epsilon\right]$, where $\epsilon \geq 0$, is sufficient for accurate image reconstruction. For $\epsilon=0$, we refer to data function $g\left(\vec{r}_{0}, \bar{t}\right)$ as a "half-time" data function, which is a minimally complete data function. We have demonstrated [2] that half-time TAT reconstruction methods can possess better statistical properties and reduced artifact levels than images reconstructed by use of conventional (i.e., full-time) methods that utilize the complete, untruncated, data function. However, truncation (with respect to $\bar{t}$ ) of the data function $g\left(\vec{r}_{0}, \bar{t}\right)$ can result in image artifacts when data inconsistencies are present. The data inconsistencies can be caused by stochastic noise introduced by the measurement process or deterministic errors created by inaccuracies in the imaging model. The image artifacts can be attributed to numerical (e.g., interpolation) errors and to nonuniform data averaging effects. To mitigate these artifacts, we propose weighted EM algorithms for image reconstruction that are described below. 


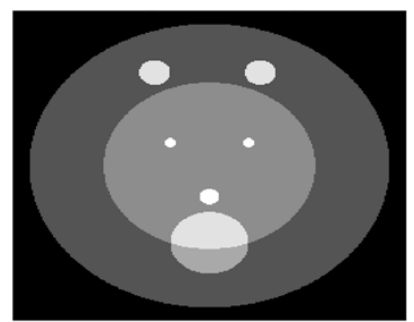

(a)

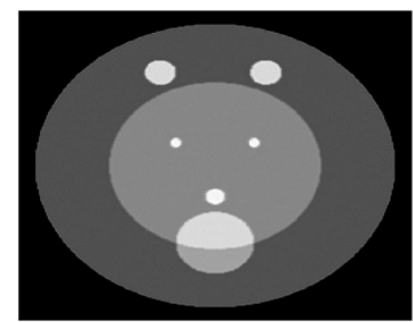

(b)

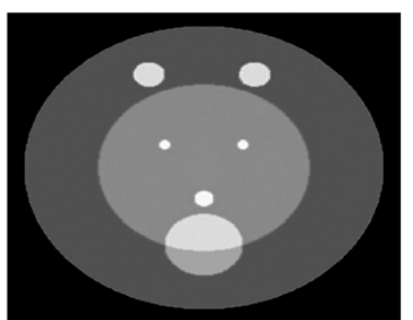

(c)

Fig. 1. (a) Numerical phantom. (b) and (c) Noiseless images reconstructed by use of weighted EM algorithms specified by weighting functions $w_{1}\left(\vec{r}_{0}, \bar{t}\right)$ and $w_{2}\left(\vec{r}_{0}, \bar{t}\right)$, respectively.

\section{Weighted Half-Time EM ALGORITHMS}

\section{A. A Family of Weighted EM Algorithms}

We will express the imaging model in (2) as

$$
g\left(\vec{r}_{0}, \bar{t}\right)=H A(\vec{r})
$$

where $H$ represents the spherical Radon transform operator, and the data function $g\left(\vec{r}_{0}, \bar{t}\right)$ and absorption function $A(\vec{r})$ are elements of the Hilbert spaces $Y$ and $X$, respectively, which are equipped with $L^{2}$ inner products. The EM algorithm can be employed for the reconstruction of $A(\vec{r})$ from knowledge of the TAT (full- or half-time) data function $g\left(\vec{r}_{0}, \bar{t}\right)$ as [3]

$$
A(\vec{r}) \equiv H^{-1} g\left(\vec{r}_{0}, \bar{t}\right)=\lim _{k \rightarrow \infty} A^{k}(\vec{r})
$$

where $H^{-1}$ is the inverse of $H$ and the iterates $A^{k}$ are defined by

$$
A^{k+1}=A^{k} \frac{H^{*}\left(\frac{g}{H A^{k}}\right)}{H^{*} m}
$$

and $k$ is a nonnegative integer. In (4), $H^{*}$ represents the adjoint operator corresponding to $H$ and the data mask $m \in Y$ satisfies $m\left(\vec{r}_{0}, \bar{t}\right)=1$ if $g\left(\vec{r}_{0}, \bar{t}\right)>0$ and zero otherwise. The adjoint operator $H^{*}$ backprojects the (filtered) data onto concentric spherical surfaces of radii $\bar{t}$ that are centered at the receiver location $\overrightarrow{r_{0}}$.

Truncation of the data function $g\left(\vec{r}_{0}, \bar{t}\right)$ can result in image artifacts when data inconsistencies are present. To mitigate these artifacts, we propose the use of a weighted EM algorithm for image reconstruction. Consider an operator $L: Y \rightarrow Y$ that is defined as

$$
L g\left(\vec{r}_{0}, \bar{t}\right) \equiv g\left(\vec{r}_{0}, \bar{t}\right) w\left(\vec{r}_{0}, \bar{t}\right),
$$

where $w\left(\overrightarrow{r_{0}}, \bar{t}\right)$ is a smooth (continuously differentiable) function on $\Omega_{0} \times\left(0,\left|\vec{r}_{0}\right|+\epsilon\right]$ that satisfies $w\left(\overrightarrow{r_{0}}, \bar{t}\right)>0$ almost everywhere ${ }^{1}$. We can obtain a weighted imaging model by acting the operator $L$ on both sides of (3) yielding

$$
g_{w}\left(\vec{r}_{0}, \bar{t}\right)=H_{w} A(\vec{r})
$$

where $g_{w}\left(\vec{r}_{0}, \bar{t}\right) \equiv g\left(\vec{r}_{0}, \bar{t}\right) w\left(\vec{r}_{0}, \bar{t}\right)$ and $H_{w} \equiv L H$. Equation (6) describes a positive integral equation. Moreover, it possesses a unique solution because (3) possess a unique solution and $L$ is a one-to-one (injective) operator on the space $Y$. Accordingly, the EM algorithm can be employed in a principled way [9] for reconstruction of $A(\vec{r})$ via inversion of (6). It can be shown readily that $H_{w}^{*}=H^{*} L$, which indicates that $H_{w}^{*}$ represents a spherical backprojection of the weighted

${ }^{1}$ More specifically, $w\left(\overrightarrow{r_{0}}, \bar{t}\right)>0$ everywhere on $\Omega_{0} \times\left(0,\left|\vec{r}_{0}\right|+\epsilon\right]$ except on a subset of measure zero.

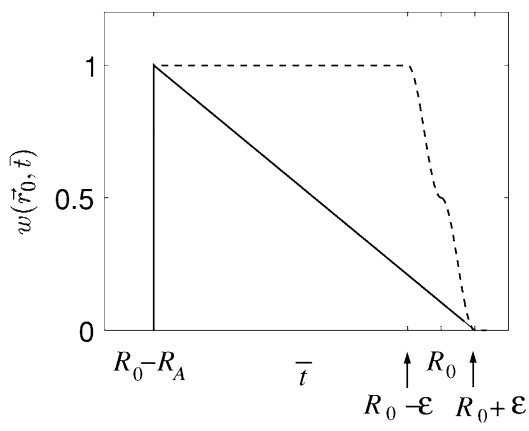

Fig. 2. The weighting function $w_{1}\left(\vec{r}_{0}, \bar{t}\right)$ (solid line) and $w_{2}\left(\vec{r}_{0}, \bar{t}\right)$ (dashed line) as defined by (9) and (10), respectively. In this example, $\epsilon=15, R_{A}=$ 128 , and $R_{0} \equiv\left|\vec{r}_{0}\right|=1.2 R_{A}$.

data function. The reconstruction of $A(\vec{r})$ by use of a weighted EM algorithm can be accomplished as

$$
A(\vec{r}) \equiv H_{w}^{-1} g_{w}\left(\vec{r}_{0}, \bar{t}\right)=\lim _{k \rightarrow \infty} A_{w}^{k}(\vec{r})
$$

where $H_{w}^{-1}$ is the inverse of $H_{w}$ and the iterates $A_{w}^{k}$ are defined by

$$
A_{w}^{k+1}=A_{w}^{k} \frac{H^{*} L\left(\frac{g_{w}}{L H A_{k}^{w}}\right)}{H^{*} L(m)}=A_{w}^{k} \frac{H^{*} L\left(\frac{g}{H A_{w}^{k}}\right)}{H^{*} L(m)}
$$

where the mask function $m$ is the same as in the conventional formula in (4).

Because there exists an infinite number of weighting functions $w\left(\vec{r}_{0}, \bar{t}\right)$, and consequently imaging models described by (6), (8) represents an infinite family of reconstruction algorithms for determination of $A(\vec{r})$. These reconstruction algorithms are equivalent mathematically, but can be expected to propagate data inconsistencies and finite sampling effects distinctively. The images reconstructed by use of different reconstruction algorithms can contain distinct artifacts due to acoustic heterogeneities and have different statistical properties. This observation presents the opportunity for the specification of a weighting function $w\left(\overrightarrow{r_{0}}, \bar{t}\right)$ (equivalently, imaging model $H_{w}$ ) that produces a reconstructed image that is optimal in a prescribed sense. It is mathematically interesting to note that the family of weighted EM algorithms described by (8) can be obtained naturally by considering the data space $Y$ to be a weighted Hilbert space [10] that employs $w\left(\vec{r}_{0}, \bar{t}\right)$ in the definition of its inner product.

\section{B. Application to Temporally Truncated TAT Data}

In this correspondence, our primary intent is to demonstrate that suitable choices for $w\left(\vec{r}_{0}, \bar{t}\right)$ can mitigate image artifacts that are attributable to temporal truncation of the TAT data function. We restrict our attention to data functions $g\left(\overrightarrow{r_{0}}, \bar{t}\right)$ that are known $\forall \vec{r}_{0} \in \Omega_{0}$ and $\forall \bar{t} \in\left(0, \vec{r}_{0}+\epsilon\right], \epsilon \geq 0$. As discussed previously, knowledge of such 


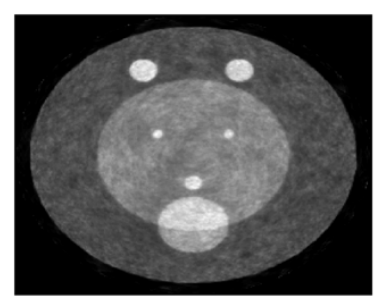

(a)

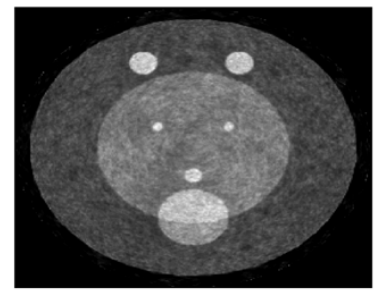

(d)

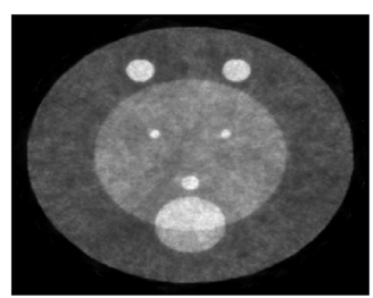

(b)

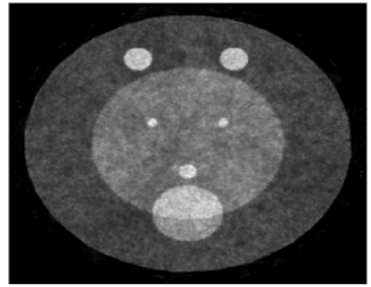

(e)

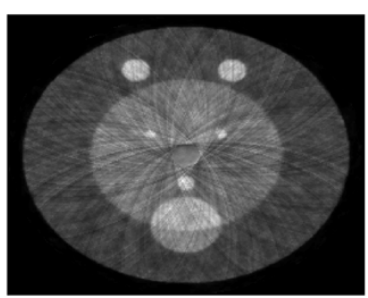

(c)

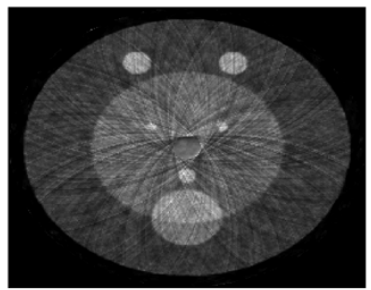

(f)

Fig. 3. Noisy images reconstructed by use of 60 iterations of the EM algorithm with weighting functions (a) $w_{1}\left(\vec{r}_{0}, \bar{t}\right),(\mathrm{b}) w_{2}\left(\vec{r}_{0}, \bar{t}\right)$, and (c) no weighting (conventional EM). (d)-(f) Corresponding images for 120 iterations of the EM algorithms.

truncated data functions is sufficient for unique and stable image reconstruction. We will require that $w\left(\overrightarrow{r_{0}}, \bar{t}\right)$ satisfies

$$
w\left(\vec{r}_{0}, \bar{t}=\left|\vec{r}_{0}\right|+\epsilon\right)=0
$$

i.e., the weighting function will be zero at the location of the data truncation in $g\left(\vec{r}_{0}, \bar{t}\right)$. Therefore, because $w\left(\vec{r}_{0}, \bar{t}\right)$ is a smooth function of $\bar{t}$, the discontinuity in $g\left(\vec{r}_{0}, \bar{t}\right)$ caused by data truncation will not be present in the weighted data function $g_{w}\left(\vec{r}_{0}, \bar{t}\right)$. Consequently, the image reconstructed from $g_{w}\left(\vec{r}_{0}, \bar{t}\right)$ can be expected to contain fewer and/or weaker artifacts than one reconstructed directly from $g\left(\vec{r}_{0}, \bar{t}\right)$. [Here, the artifacts that we refer to are the ones that can be attributed to the data truncation in $g\left(\vec{r}_{0}, \bar{t}\right)$.] In the following sections, we suggest and demonstrate the use of several choices of $w\left(\vec{r}_{0}, \bar{t}\right)$.

Note that the strategy of employing a weighting function to eliminate discontinuities in truncated tomographic data is well known. For example, this approach is used commonly in the truncated X-ray fan-beam and cone-beam tomography problems [11], [12] that utilize analytic inversion formulas. However, in these applications, the weighting function serves to both eliminate the data discontinuity and properly normalize redundant information contributed by the overscan. The weighting function in our EM algorithm serves only to accomplish the former objective; the normalization of the measurement data is accomplished implicitly by the EM algorithm [see the denominator of (8)]. This feature of the EM algorithm is particularly convenient for our purposes because the explicit structure of data redundancies in TAT is not known at the present time.

\section{NUMERICAL STUDIES}

We performed numerical studies using simulation and experimental TAT measurement data to investigate the weighted EM-based reconstruction approaches for TAT. Although both the simulation and experimental data corresponded to 2-D TAT measurement geometries, the conclusions derived from these studies are also applicable to the 3-D case.

The 2-D numerical phantom shown in Fig. 1(a) was chosen to represent the absorption function $A(\vec{r})$. From knowledge of $A(\vec{r})$, which had a radius of $R_{A}=1$ (arbitrary units), a truncated data function was calculated numerically assuming the the 2-D measurement geometry described in [2] with $\left|\vec{r}_{0}\right|=1.2$. The data function was determined at 360 equally spaced positions on the circular aperture and at 138 equally spaced values of $\bar{t}$ over the interval $\left[\left|\vec{r}_{0}\right|-R_{A},\left|\vec{r}_{0}\right|+\epsilon\right]$. The overscan parameter $\epsilon$ satisfied $\left(\epsilon / R_{A}\right)=(10 / 128)$. Noisy versions of the simulated data functions were created by treating the pressure signal $p\left(\vec{r}_{0}, t_{0}\right)$ as an uncorrelated Gaussian random process with a constant variance of $\sigma^{2}=(0.03)^{2}$, and integrating the pressure signal according to (2). An explicit description of this noise model is provided by (23) with $a=0$ in [2]. The two experimental TAT data sets described in [2] were employed also. From the experimental data, truncated data functions were defined that corresponded to overscan parameters satisfying $\left(\epsilon / R_{A}\right)=(50 / 952)$ (for the phantom object) and $\left(\epsilon / R_{A}\right)=(50 / 500)$ (for the mouse brain).

Images were reconstructed by use of the conventional EM algorithm and two versions of the weighted EM algorithm. The weighted EM algorithms were specified by the weighting functions

$$
\begin{aligned}
& w_{1}\left(\vec{r}_{0}, \bar{t}\right) \\
& \quad= \begin{cases}\frac{\left|\vec{r}_{0}\right|+\epsilon-\bar{t}}{R_{A}+\epsilon}: & \left|\vec{r}_{0}\right|-R_{A} \leq \bar{t} \leq\left|\vec{r}_{0}\right|+\epsilon \\
0: & \text { otherwise }\end{cases} \\
& \text { and } \\
& \quad w_{2}\left(\vec{r}_{0}, \bar{t}\right) \\
& \quad= \begin{cases}1: & \left|\vec{r}_{0}\right|-R_{A} \leq \bar{t} \leq\left|\vec{r}_{0}\right|-\epsilon \\
0.5+0.5 \sin ^{2}\left[\frac{\pi}{2 \epsilon}\left(\left|\vec{r}_{0}\right|-\bar{t}\right)\right]: & \left|\vec{r}_{0}\right|-\epsilon<\bar{t} \leq\left|\vec{r}_{0}\right| \\
0.5-0.5 \sin ^{2}\left[\frac{\pi}{2 \epsilon}\left(\left|\vec{r}_{0}\right|-\bar{t}\right)\right]: & \left|\vec{r}_{0}\right|<\bar{t} \leq\left|\vec{r}_{0}\right|+\epsilon .\end{cases}
\end{aligned}
$$

Plots of $w_{1}\left(\vec{r}_{0}, \bar{t}\right)$ and $w_{2}\left(\vec{r}_{0}, \bar{t}\right)$ are shown in Fig. 2 . The weighting function $w_{1}$ is a linear function that decreases from 1 to 0 along $\bar{t}$ on the truncated data range. The weighting function $w_{2}$ was proposed previously in the context of asymmetric fan-beam tomography [11]. Note that the first-order derivative of $w_{2}$ extends continuously to the data truncation point $\bar{t}=\left|\vec{r}_{0}\right|+\epsilon$.

\section{RECONSTRUCTED IMAGES}

Fig. 1(b) and (c) displays the images reconstructed from the noiseless simulated data by use of the weighted EM algorithms specified by $w_{1}\left(\vec{r}_{0}, \bar{t}\right)$ and $w_{2}\left(\vec{r}_{0}, \bar{t}\right)$, respectively. As expected, both algorithms yielded nearly identical reconstructions that accurately represented the true absorption function $A(\vec{r})$. Although not shown, the image reconstructed by use of the conventional EM algorithm is also virtually identical to those shown in Fig. 1. These results corroborate our claim 


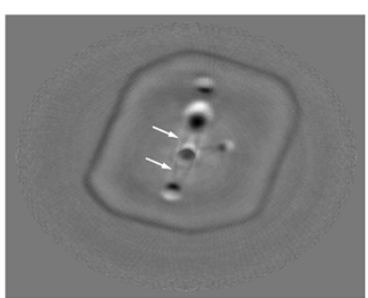

(a)

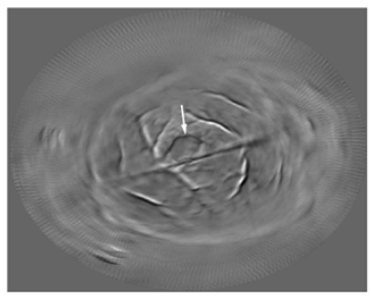

(c)

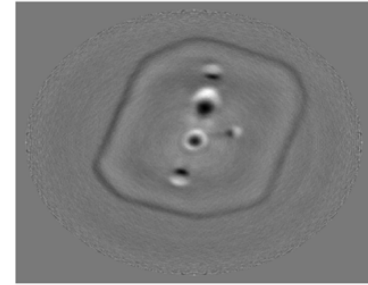

(b)

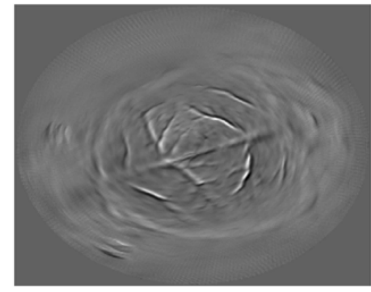

(d)
Fig. 4. (a) and (c) Images reconstructed from experimental TAT data by use of the conventional (unweighted) EM algorithm. (b) and (d) By use of the weighted EM algorithm with weighting function $w_{1}\left(\vec{r}_{0}, \bar{t}\right)$. The arrows in (a) and (c) indicate the position of artifacts that are not present in (b) and (d). In all cases, the EM algorithms were terminated at 100 iterations. Arrows in (a) and (c) indicate artifacts near the center of reconstructed images.

that the members of the infinite family of weighted EM algorithms are equivalent to the conventional EM algorithm and exact mathematically.

Fig. 3(a)-(c) displays images reconstructed from the noisy simulation data by use of 60 iterations of the EM algorithms with weighting functions $w_{1}\left(\vec{r}_{0}, \bar{t}\right), w_{2}\left(\vec{r}_{0}, \bar{t}\right)$ and no weighting (conventional EM), respectively. The noise patterns of the images in Fig. 3(a) and (b) is distinct, which is consistent with our claim that the members of the infinite family of weighted EM algorithms will generally propagate data inconsistencies in different ways. Note that both of these images have a less noisy appearance than the image reconstructed by use of the conventional EM algorithm that is shown in Fig. 3(c). The image reconstructed by use of the conventional EM algorithm contains circular arc-like artifacts that are attributable to the abrupt truncation of the data function. Additionally, a circular disk artifact is present at the center of the image. The radius of this artifact is equal to the length of the overscan $(\epsilon)$ in the data function. For an exactly half-time data function, the disk artifact reduces to a bright point in the center of image space. Fig. 3(d)-(f) shows the corresponding images obtained by use 120 iterations of the EM algorithms.

Fig. 4(a) and (c) displays images reconstructed from the experimental data sets by use of the conventional EM algorithm. The corresponding images reconstructed by use of the weighted EM algorithm specified by $w_{1}\left(\vec{r}_{0}, \bar{t}\right)$ are displayed in Fig. 4(b) and (d). For both objects, the weighted EM algorithm produced images that contained fewer and weaker artifacts than produced by the conventional EM algorithm.

\section{SUMMARY}

In this correspondence, we proposed and investigated an infinite family of weighted expectation maximization (EM) algorithms for reconstruction of TAT images. The weighted EM algorithms were shown to be equivalent mathematically to the conventional EM algorithm, but generally propagated data inconsistencies in different ways. We demonstrated that suitable choices of the weighted EM algorithm could mitigate image artifacts that are attributable to temporal truncation of the TAT data function.

Although the use of weighting functions in analytic tomographic reconstruction approaches is well known, it appears that little or no work has been devoted to investigating their use in iterative reconstruction approaches. The flexibility afforded by the weighted EM reconstruction approach permits the specification of a reconstruction algorithm that produces an image that is optimal in a prescribed sense. For example, in addition to reducing image artifacts attributable to data truncation, the weighting function can (in principle) be designed to reduce optimally the statistical variance of the reconstructed image for a given data noise model. The exploration of this idea and the investigation of other characteristics of the weighted EM reconstruction algorithms represent topics for future work.

\section{ACKNOWLEDGMENT}

The authors would like to thank Dr. G. Ku for his role in acquiring the experimental TAT data sets used in this paper.

\section{REFERENCES}

[1] G. Ku, X. Wang, G. Stoica, and L. V. Wang, "Multiple-bandwidth photoacoustic tomography," Phys. Med. Biol., vol. 49, pp. 1329-1338, 2004.

[2] M. A. Anastasio, J. Zhang, X. Pan, Y. Zou, G. Keng, and L. V. Wang, "Half-time image reconstruction in thermoacoustic tomography," IEEE Trans. Med. Imag., vol. 24, no. 2, pp. 199-210, Feb. 2005.

[3] X. Pan, Y. Zou, and M. A. Anastasio, "Data redundancy and reduced-scan reconstruction in reflectivity tomography," IEEE Trans. Image Process., vol. 12, no. 7, pp. 784-795, Jul. 2003.

[4] M. Xu and L. V. Wang, "Time-domain reconstruction for thermoacoustic tomography in a spherical geometry," IEEE Trans. Med. Imag., vol. 21, no. 7, pp. 814-822, Jul. 2002

[5] M. Xu, Y. Xu, and L. V. Wang, "Time-domain reconstruction algorithms and numerical simulations for thermoacoustic tomography in various geometries," IEEE Trans. Biomed. Eng., vol. 50, no. 9, pp. 1086-1099, Sep. 2003.

[6] S. Norton, "Reconstruction of a two-dimensional reflecting medium over a circular domain: Exact solution," J. Acoust. Soc. Amer., vol. 67, no. 4, pp. 1266-1273, 1980.

[7] S. Norton and M. Linzer, "Ultrasonic reflectivity imaging in three dimensions: Exact inverse scattering solutions for plane, cylindrical, and spherical apertures," IEEE Trans. Biomed. Eng., vol. BME-28, pp. 202-220, 1981.

[8] D. Finch, S. Patch, and S. Rakesh, "Determining a function from its mean values over a family of spheres," in SIAM J. Math. Anal., vol. 35, 2004, pp. 1213-1240.

[9] Y. Vardi and D. Lee, "From image deblurring to optimal investments: Maximum likelihood solutions for positive linear inverse problems," $J$. Roy. Statist. Soc. B, vol. 55, pp. 569-612, 1993.

[10] M. Bertero and P. Boccacci, Inverse Problems in Imaging. Bristol, MA: Inst. Phys., 1998.

[11] P. La Riviere, X. Pan, D. Gilland, C. M. Kao, W. Chang, and R. Jaszczak, "Transmission image reconstruction and redundant information in SPECT with asymmetric fanbeam collimation," IEEE Trans. Nucl. Sci., vol. 48, no. 4, pp. 1357-1363, Aug. 2001.

[12] P. Cho, R. Johnson, and T. Griffin, "Cone-beam CT for radiotherapy applications," Phys. Med. Biol., vol. 40, pp. 1863-1883, 1995. 